\title{
Peritumoral ductular reaction can be a prognostic factor for intrahepatic cholangiocarcinoma
}

\author{
Zhenyang Shen ${ }^{1 \dagger}$, Jingbo Xiao ${ }^{1 \dagger}$, Junjun Wang ${ }^{1}$, Lungen $\mathrm{Lu}^{1}$, Xinjian $\mathrm{Wan}^{2}$ and Xiaobo Cai ${ }^{{ }^{*}}$
}

\begin{abstract}
Background: Peritumoral ductular reaction (DR) was reported to be related to the prognosis of combined hepatocellular-cholangiocarcinoma and hepatocellular carcinoma. Non-mucin-producing intrahepatic cholangiocarcinoma (ICC) which may be derived from small bile duct cells or liver progenitor cells (LPCS) was known to us. However, whether peritumoral DR is also related to non-mucin-producing ICCs remains to be investigated.
\end{abstract}

Methods: Forty-seven patients with non-mucin-producing ICC were eventually included in the study and clinicopathological variables were collected. Immunohistochemical analysis and immunofluorescence staining for cytokeratin 19, proliferating cell nuclear antigen, and a-smooth muscle actin were performed in tumor and peritumor liver tissues.

Results: A significant correlation existed between peritumoral DR and local inflammation and fibrosis. ( $r=0.357$, $95 \% \mathrm{Cl}, 0.037-0.557 ; P=0.008$ and $r=0.742,95 \% \mathrm{Cl}, 0.580-0.849 ; P<0.001$, respectively). Patients with obvious peritumoral DR had high recurrence rate $(81.8 \%$ vs $56.0 \%, P=0.058)$ and poor overall and disease-free survival time ( $P=0.01$ and $P=0.03$, respectively) comparing with mild peritumoral DR. Compared with the mild peritumoral DR group, the proliferation activity of LPCs/ cholangiocytes was higher in obvious peritumoral DR, which, however, was not statistically significant. ( $0.43 \pm 0.29$ vs $0.28 \pm 0.31, P=0.172)$. Furthermore, the correlation analysis showed that the DR grade was positively related to the portal/septala-SMA level $(r=0.359, P=0.001)$.

Conclusions: Peritumoral DR was associated with local inflammation and fibrosis. Patients with non-mucinproducing ICC having obvious peritumoral DR had a poor prognosis. Peritumoral DR could be a prognostic factor for ICC. However, the mechanism should be further investigated.

Keywords: Peritumoral, Ductular reaction, Prognosis, Intrahepatic cholangiocarcinoma

\footnotetext{
* Correspondence: caixiaobo1979@hotmail.com

'Zhenyang Shen and Jingbo Xiao contributed equally to this work.

${ }^{1}$ Department of Gastroenterology, Shanghai General Hospital, Shanghai Jiao

Tong University School of Medicine, Shanghai, China

Full list of author information is available at the end of the article
}

(C) The Author(s). 2020 Open Access This article is licensed under a Creative Commons Attribution 4.0 International License, which permits use, sharing, adaptation, distribution and reproduction in any medium or format, as long as you give appropriate credit to the original author(s) and the source, provide a link to the Creative Commons licence, and indicate if changes were made. The images or other third party material in this article are included in the article's Creative Commons licence, unless indicated otherwise in a credit line to the material. If material is not included in the article's Creative Commons licence and your intended use is not permitted by statutory regulation or exceeds the permitted use, you will need to obtain permission directly from the copyright holder. To view a copy of this licence, visit http://creativecommons.org/licenses/by/4.0/ The Creative Commons Public Domain Dedication waiver (http://creativecommons.org/publicdomain/zero/1.0/) applies to the data made available in this article, unless otherwise stated in a credit line to the data. 


\section{Background}

Cholangiocarcinoma (CCA) is highly malignant with a 5year survival rate of 0 to $10 \%$ [1]. CCA can be divided mainly into extrahepatic cholangiocarcinoma and intrahepatic cholangiocarcinoma (ICC) based on the anatomic features [2]. ICC accounts for approximate $10 \%$ of primary liver cancer, and the incidence continues to increase in recent years [3]. ICC can also be divided into two categories: mucin producing and non-mucin producing, which have different pathological characteristics and origins. Although ICC is thought to develop from the intrahepatic bile duct, at least some ICCs, especially non-mucin-producing ICCs, may be derived from small bile duct cells or liver progenitor cells (LPCs) [4, 5]. In combined hepatocellularcholangiocarcinoma may originate from bipotential LPC due to their dual characteristics of cholangiocytes and hepatocytes, background LPC was reported to be a prognostic factor after resection due to the origin of tumor cells [6]. Ductular reaction (DR) consisting of LPCs or small cholangiocytes represents hepatic or biliary regeneration in the liver. Peritumoral DR consisting of LPCs or small cholangiocytes is related to the prognosis of combined hepatocellular-cholangiocarcinoma and hepatocellular carcinoma [6, 7]. However, whether similar finding can also be observed in non-mucin-producing ICC remains to be investigated.

In the present study, patients with non-mucinproducing ICC were included, and the extent of peritumoral DR was evaluated to explore whether a relationship existed between DR and prognosis of ICC as well as its potential mechanism.

\section{Methods}

\section{Patients and specimens}

From January 2004 to December 2016, 47 patients with ICC, who underwent curative surgery in Shanghai General Hospital, Shanghai Jiao Tong University School of Medicine were included in the study. The written informed consent was obtained from each patient under a protocol approved by the ethics committee of Shanghai General Hospital. The tumor stage was determined according to the 2009 UICC TNM classification system [8]. The tumor and peritumor $(<2 \mathrm{~cm}$ away from the tumor) tissues from each patient and the available nontumor tissues ( $>2 \mathrm{~cm}$ away from the tumor) from $30 \mathrm{pa}$ tients were paraffin embedded.

\section{Histological and immunohistochemical analysis}

Liver tissues were fixed in $4 \%$ formaldehyde and embedded in paraffin. Sections ( $4 \mu \mathrm{m}$ thick) were stained with hematoxylin and eosin stain. According to the Scheuer scoring system, the inflammation and fibrosis of the peritumor tissues were independently scored by two experienced pathologists. If the scores of two pathologists were inconsistent, we had asked the third experienced pathologist to score and made the decision [9]. For immunohistochemical analysis, having been washed with phosphate-buffered saline, the sections were transferred into $10 \mathrm{mM}$ sodium citrate buffer ( $\mathrm{pH}$ 6.0), and antigen unmasking was performed in a microwave. After cooling down, the sections were incubated with peroxidase blocking reagent (Dako, Hamburg, Germany) for $1 \mathrm{~h}$ and then stained overnight at $4{ }^{\circ} \mathrm{C}$ with the following primary antibodies: anti-cytokeratin 19 (CK19) (Dako), 1:200; anti-proliferating cell nuclear antigen (PCNA) (Santa Cruz Biotechnology, USA), 1:200. The sections were developed with diaminobenzidine for 5 mins. The DR grade was evaluated according to the following standards: 0 , no or minimal DR around a few portal tracts and septa; 1, focal DR around most portal tracts/septa; 2 , continuous DR around $<30 \%$ of portal tracts/septa; 3 , continuous DR around $30-50 \%$ of portal tracts/septa; and 4, continuous DR around more than $50 \%$ of portal tracts/septa (Fig. 1). For quantitative analysis, mean values of immunoreactive cells by counting the three fields including the portal or septal area at $200 \times$ magnification were randomly obtained. The proliferation index (PI) was calculated as the ratio between the number of $\mathrm{PCNA}^{+}$cells and the total number of reactive ductular cells or tumor cells. The high PI means that the ratio of the number of $\mathrm{PCNA}^{+}$cells in number of reactive ductular cells or tumor cells is $>50 \%$.

\section{Double-fluorescence immunostaining}

Double-fluorescence immunostaining of formalin-fixed and paraffin-embedded tissue was performed using a sequential fluorescence method as previously described [10]. Alexa488 or Alexa647-conjugated goat antirabbit antibody (Invitrogen) was used as the secondary antibody. Nuclei were stained with 4',6-diamidino-2-phenylindole (DAPI). Immunofluorescence was observed using Olympus IX-71 inverted microscope.

\section{Statistical analysis}

Data were analyzed using SPSS version 23.0 for Windows (SPSS Inc., Chicago, IL, USA) and presented as means and standard deviations $( \pm \mathrm{SD})$. Student $t$ tests were used to compare the continuous quantitative data. A two-tailed Wilcoxon signed-rank test was used to compare ranked variables. The correlation between the degree of DR and clinicopathological variables was determined by Spearman or Pearson correlation as appropriate. The overall survival and disease-free survival were analyzed via the Kaplan-Meier method and compared using the log rank test. Multivariate Cox proportional hazard regression analyses were performed to identify risk factors for overall survival and disease-free 

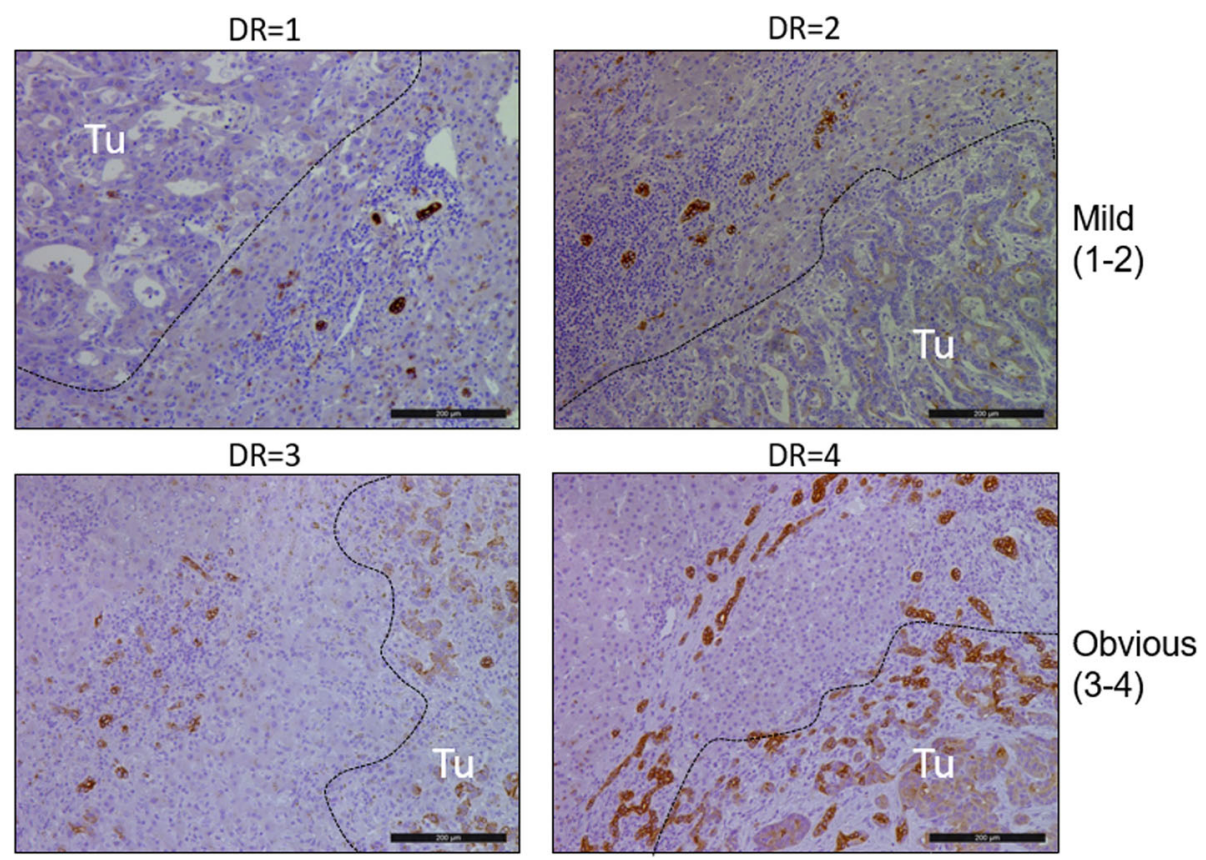

Fig. 1 Representative figures showing different grades of peritumoral DR indicated by immunohistochemical staining for CK19 in ICC (scale bar $=200 \mu \mathrm{m}$ )

survival. $P$ value $<0.05$ was considered statistically significant.

\section{Results}

\section{Clinicopathological and follow-up data}

Forty-seven patients (30 men and 17 women) were eventually included in the present study, with a mean age of $58.4 \pm 11.0$ years. Nine patients had lymph node invasion, and five patients had distant metastasis at the time of diagnosis. After a follow-up period of $25.7 \pm 19.1$ months, 32 patients $(68.1 \%)$ developed intrahepatic recurrence after surgery (Table 1).

\section{Peritumoral DR was related to local inflammation and fibrosis}

The correlation analysis showed that peritumoral DR was significantly correlated with local inflammation and fibrosis $(r=0.357,95 \% \mathrm{CI}, 0.037-0.557 ; P=0.008$ and $r=0.742$, 95\% CI, 0.580-0.849; $P<0.001$, respectively) (Fig. 2). Due to the different definition of background $\mathrm{DR}$, the DR grade in peritumor $(<2 \mathrm{~cm}$ away from the tumor) and nontumor ( $>2 \mathrm{~cm}$ away from the tumor) areas was also compared in 30 patients whose liver tissues from both sites were available. The results demonstrated a small difference in local inflammation and fibrosis between them, but with no statistical significance. The DR grade in nontumor area is positively correlated with that in peritumoral area $(r=0.713,95 \% \mathrm{CI}$, 0.499-0.862; $P<0.001$ ) (Fig. 3). These results indicated a
Table 1 The comparison of clinicopathological parameters between mild and obvious peritumoral DR patients

\begin{tabular}{|c|c|c|c|}
\hline & Mild DR $(n=25)$ & Obvious DR $(n=22)$ & $P$ value \\
\hline Age & $59.3 \pm 9.4$ & $57.4 \pm 12.8$ & 0.489 \\
\hline Gender & & & 0.560 \\
\hline Male & $15(60.0 \%)$ & $15(68.1 \%)$ & \\
\hline Female & $10(40.0 \%)$ & 7 (31.9\%) & \\
\hline TNM stages & & & 0.724 \\
\hline$|-| \mid$ & $8(32.0 \%)$ & $6(27.3 \%)$ & \\
\hline III-IV & 17 (68.0\%) & $16(72.7 \%)$ & \\
\hline $\mathrm{T}$ & & & 0.491 \\
\hline $1-2$ & $10(40.0 \%)$ & $11(50.0 \%)$ & \\
\hline $3-4$ & $15(60.0 \%)$ & $11(50.0 \%)$ & \\
\hline N & & & 0.559 \\
\hline 0 & $21(84.0 \%)$ & $17(77.3 \%)$ & \\
\hline 1 & $4(16.0 \%)$ & $5(22.7 \%)$ & \\
\hline M & & & 0.532 \\
\hline 0 & $23(92.0 \%)$ & 19 (86.4\%) & \\
\hline 1 & $2(8.0 \%)$ & $3(13.6 \%)$ & \\
\hline Differentiation & & & 0.510 \\
\hline $1-2$ & $16(64.0 \%)$ & $12(54.5 \%)$ & \\
\hline $3-4$ & $9(36.0 \%)$ & $10(45.5 \%)$ & \\
\hline Recurrence & & & 0.057 \\
\hline No & $11(44.0 \%)$ & $4(18.2 \%)$ & \\
\hline Yes & $14(56.0 \%)$ & $18(81.8 \%)$ & \\
\hline
\end{tabular}




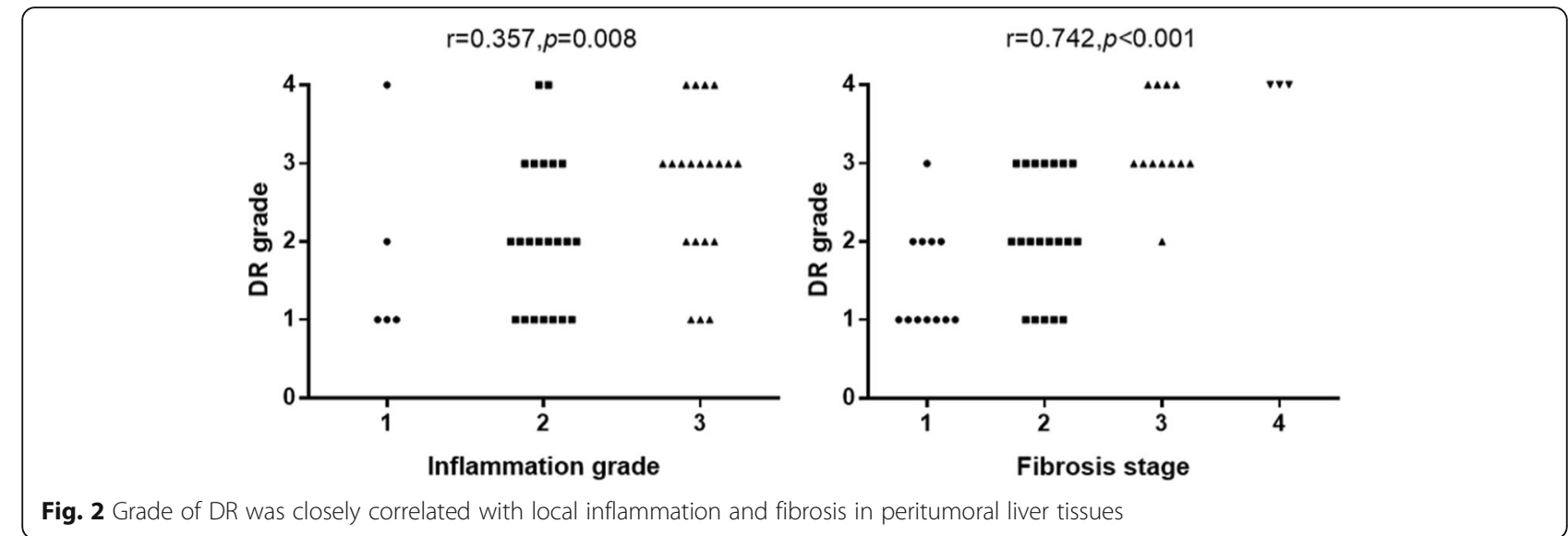

similar extent of DR and local environment between peritumor and nontumor areas.

\section{Peritumoral DR was related to the prognosis of ICC}

According to the grade of peritumoral DR, patients with ICC were divided into two groups: mild peritumoral DR (grades 1 and 2) $(n=25)$ and obvious DR (grades 3 and 4) $(n=22)$. Age, gender composition, TNM stages, and tumor differentiation were not significantly different between these two groups. However, the trend of tumor recurrence in the obvious DR group was much higher than that in the mild DR group but it was not statistically significant $(81.8 \%$ vs $56.0 \%, P=0.058)$ (Table 1$)$. The survival analysis showed that patients with obvious peritumoral DR had poor overall and disease-free survival $(P=0.01$ and $P=0.03$, respectively) (Fig. 4). In the multivariate analysis, obvious peritumoral DR was negatively associated with overall survival and disease-free survival (95\% CI, 0.0420.588 ; $P=0.016$ and $95 \% \mathrm{CI}, 0.072-0.647 ; P=0.013$, respectively) (Supplementary Table 1-2).

\section{Different proliferation of peritumoral ductular cells}

According to the previous description, PI was used to mark the extent of proliferation (Fig. 5a). The obvious peritumoral DR group showed a higher PI trend of ductular cells compared with the mild peritumoral DR group but failed to achieve statistical significance due to high variation $(0.43 \pm 0.29$ vs $0.28 \pm 0.31, P=0.172)$. The percentage of high PI (>50\%) ductular cells was also higher in the obvious peritumoral DR group $(44.44 \%$ vs $30.77 \%$, $P<0.01)$. Undoubtedly, the tumor cells showed much higher PI compared with the other two groups (Fig. 5b).

\section{Different grade of peritumor DR was related to different microenvironments}

ICC is a kind of tumor with abundant extracellular matrix (ECM), which plays an indispensable role in tumor progression. Double-fluorescence immunostaining showed the $\alpha$-smooth muscle actin ( $\alpha$-SMA)-positive fibrosis background and CK19-positive ductular and tumor cells. The results demonstrated that there were more abundant ECM and $\alpha$-SMA-positive vessels in peritumoral areas of obvious DR group than in mild DR group, which was similar to that in the tumor (Fig. 6a). The correlation analysis showed that the DR grade was positively related to the portal/septal $\alpha$-SMA level $(\mathrm{r}=$ 0.359, $P=0.001$ ) (Fig. 6b).

\section{Discussion}

ICC can pathologically be divided into two categories: mucin producing and non-mucin producing. The former is derived from large intrahepatic bile ducts and has pathological features which are similar to those of extrahepatic cholangiocarcinoma, while the latter is considered to

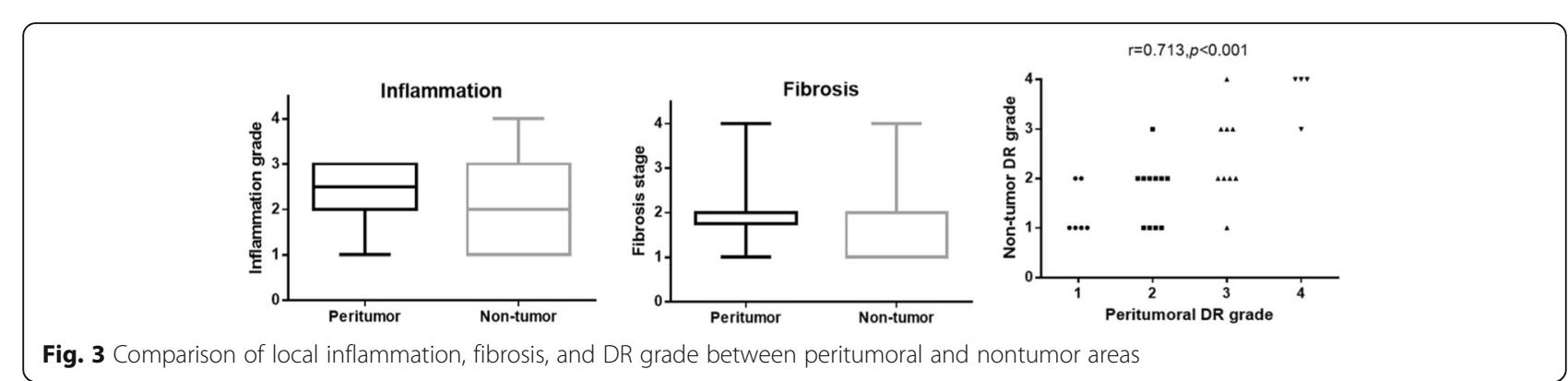



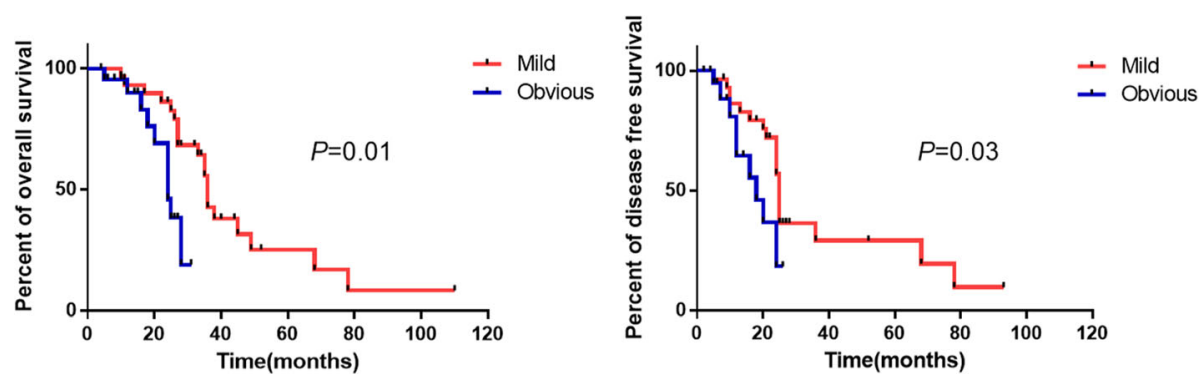

Fig. 4 Kaplan-Meier analysis of the overall and disease-free survival of patients with ICC having different grades of DR

be derived from LPCs or small bile duct cells $[4,5]$. ICC and hepatocellular carcinoma share some common risk factors, providing evidence that some ICCs might originate from bipotential LPC. A recent meta-analysis showed that cirrhosis and hepatitis B or hepatitis $\mathrm{C}$ virus (HCV) infection was a potential risk factor with the odds ratio value of 22.92, 5.1, and 4.8 respectively [11]. Another study showed that $\mathrm{HCV}$ infection and cirrhosis were the main risk factors for ICC [12]. On the contrary, liver fibrosis and inflammation were also main factors influencing the extent of DR in chronic liver diseases [13-15]. Therefore, it was speculated that peritumoral DR might be related to ICC due to common influencing factors and cell origins.
For combined hepatocellular-cholangiocarcinoma derived from LPC, the active peritumoral LPC was considered to be related to recurrence after resection [6]. Peritumoral DR was also correlated with the prognosis in hepatocellular carcinoma [7]. However, the relationship between peritumoral DR and prognosis of ICC is still not elucidated. Because non-mucin-producing ICC may be derived from small cholangiocytes or LPCs, which are the main source of DR, their relationship was studied. In the present study, the peritumoral DR was closely related to local liver inflammation and fibrosis, just like in chronic liver disease. Also, a similar extent of DR and liver inflammation or fibrosis was found in

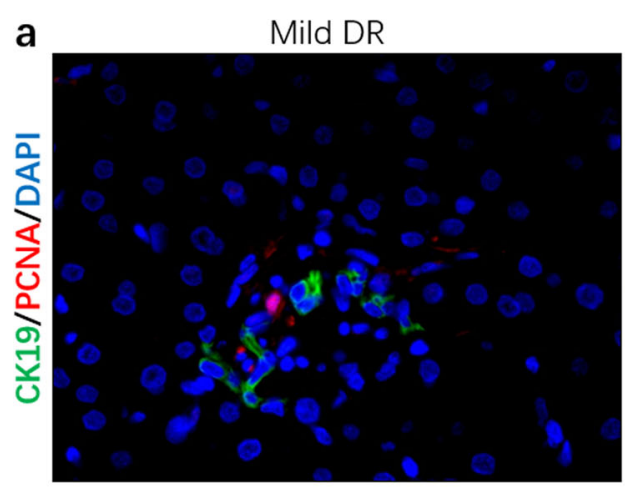

Tumor

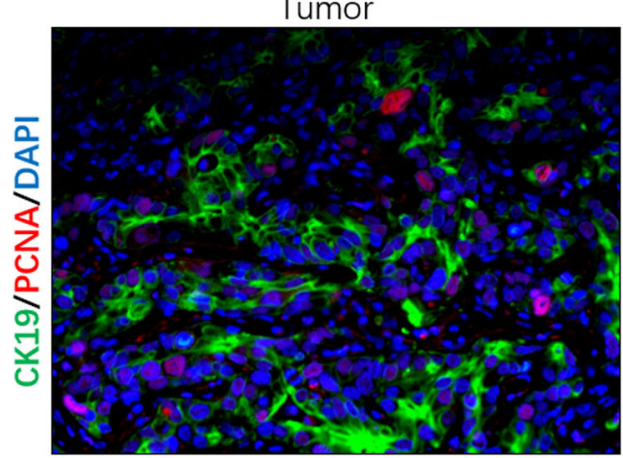

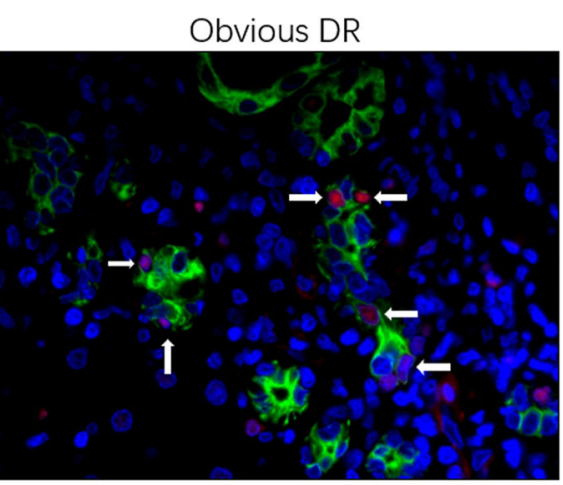

b

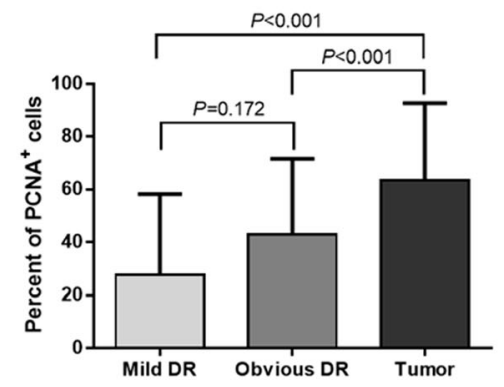

Fig. $5 \mathrm{Pl}$ in the different peritumoral DR groups and tumor group. a CK19/PCNA positive expression in the mild and obvious peritumoral DR group and tumor group. b The obvious peritumoral DR group indicated a higher PI trend of ductular cells compared with the mild peritumoral DR group, and tumor group showed much higher PI compared with the other two groups. [PI = number of PCNA-positive (arrow)/total number of reactive ductular/tumor cells] 


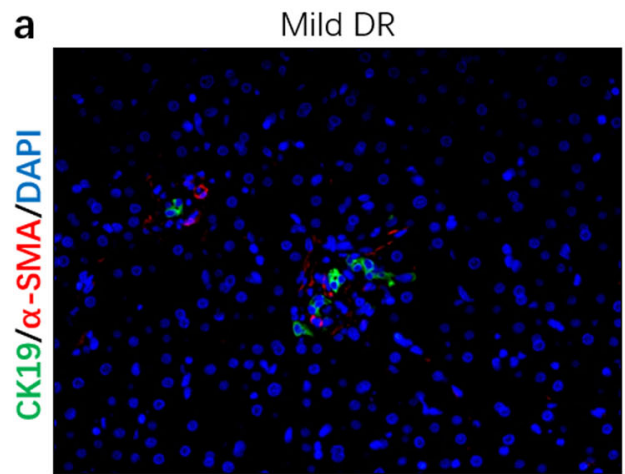

Tumor

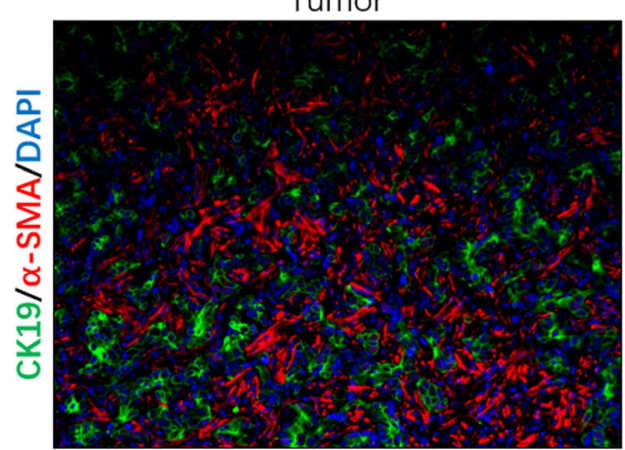

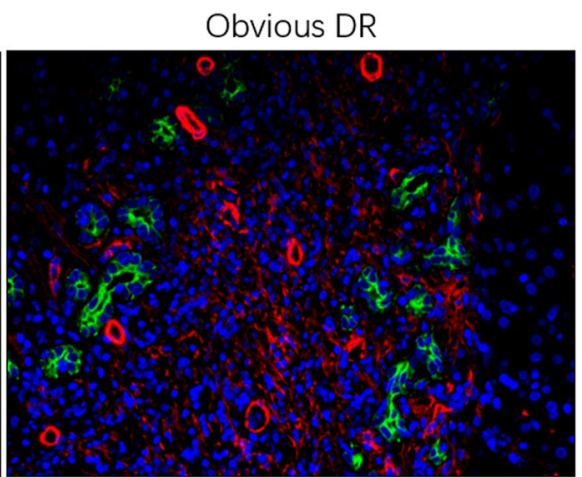

b

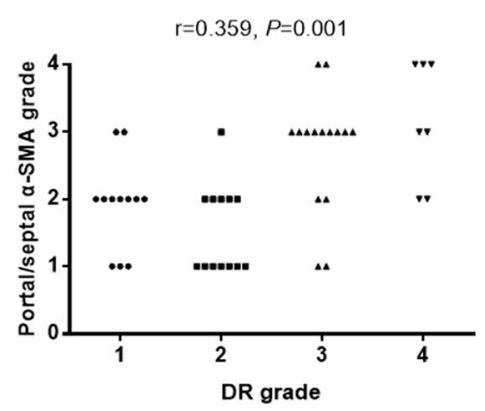

Fig. 6 Microenvironment in the different peritumoral DR groups and tumor group. a The obvious DR group had more abundant ECM and aSMA-positive vessels in peritumoral areas than in the mild DR group, and tumor group had similar microenvironment with the obvious DR group. b The correlation analysis illustrated that the DR grade was positively related to the portal/septal a-SMA level

peritumor and nontumor areas $(<2$ or $>2 \mathrm{~cm}$ from the tumor), indicating that such microenvironment was not just located in peritumoral areas.

According to the grade of peritumoral DR, patients with ICC were divided into two groups: mild DR and obvious DR groups. The clinical and pathological variables were compared between the two groups, and the results showed that the latter had a higher recurrence rate compared with the former. The survival analysis showed that patients with obvious peritumoral DR had significant poor overall and disease-free survival time. Hence, it could be concluded that peritumoral DR was related to non-mucin-producing ICC and could be a prognostic factor. Because the sample was not adequately powered to detect differences, the clinical outcomes may have been affected. Studies with larger sample sizes are warranted to confirm our results.

The mechanism underlying the relationship between peritumor DR and ICC is still not clear. A study demonstrating the correlation of peritumor DR with the prognosis of combined hepatocellular-cholangiocarcinoma speculated that peritumoral LPC probably provided the "field effect" and led to the development of tumor [6]. The present study also showed that peritumoral DR was related to ICC occurrence and poor prognosis. However, it is still hard to elucidate whether the activated peritumor LPCs/cholangiocytes could cause tumor occurrence. Immunostaining by PCNA showed that the proliferation activity of LPC was significantly enhanced in the obvious DR group than in the mild DR group. ICC is characterized by its abundant ECM, and the tumor-related macrophages and fibroblasts located in the ECM are related to poor prognosis. Immunostaining by $\alpha$-SMA also demonstrated that significantly abundant ECM and vessels were accompanied by obvious DR, indicating that obvious peritumoral DR might share similar microenvironment with ICC. To sum up, peritumoral DR offered "field effect" which is likely to affect a wide area of the target tissue. In this study, we demonstrated that obvious peritumoral DR not only shared some characteristics with ICC but also provided insight into the recurrence of ICC in patients who have undergone curative surgery.

\section{Conclusions}

The present study showed that patients with ICC having obvious peritumoral DR had a poor prognosis. Obvious peritumoral DR had a high proliferation activity of LPCs/cholangiocytes and abundant background ECM, which was similar to ICC. Although it is unclear whether activated peritumoral LPCs/ 
cholangiocytes could lead to tumor occurrence or only be the result of fibrosis, which is also a risk factor of ICC, the results suggest that peritumoral DR could be a prognostic factor for ICC. However, studies with larger sample sizes are needed to confirm our results and the mechanism should be further investigated.

\section{Supplementary information}

Supplementary information accompanies this paper at https://doi.org/10. 1186/s12876-020-01471-0.

Additional file 1: Supplementary Table 1. Multivariate analysis of overall survival. Supplementary Table 2. Multivariate analysis of disease-free survival.

\section{Abbreviations}

DR: Peritumoral ductular reaction; ICC: Intrahepatic cholangiocarcinoma; LPC: Liver progenitor cells; CK19: Cytokeratin 19; PCNA: Proliferating cell nuclear antigen; a-SMA: Alpha-smooth muscle actin; Cl: Confidence interval; CCA: Cholangiocarcinoma; UICC: Union Internationale Contre le Cancer; PI: Proliferation index; DAPI: 4',6-diamidino-2-phenylindole; SD: Standard deviations; ECM: Extracellular matrix; HCV: Hepatitis C virus; HR: Hazard ratio

\section{Acknowledgements}

We would like to thank all participants for their support in this study.

\section{Authors' contributions}

Conceived and designed the study: XBC, LGL, XJW. Collected and analyzed the data: ZYS, JBX, JJW. Interpreted the results and wrote the paper: XBC, ZYS, JBX, JJW. All authors read and approved the final manuscript

\section{Funding}

This work was supported by the National Science and Technology Major Special Project for New Drug Development (2018ZX09201016).

\section{Availability of data and materials}

The datasets generated or analyzed during the current study are available from the corresponding author on reasonable request.

\section{Ethics approval and consent to participate}

The clinical study was approved by the Ethics Committee of Shanghai General Hospital, Shanghai Jiao Tong University School of Medicine. All participants have signed the written informed consent.

\section{Consent for publication}

Not applicable.

\section{Competing interests}

The authors declare that they have no competing interests.

\section{Author details}

'Department of Gastroenterology, Shanghai General Hospital, Shanghai Jiao Tong University School of Medicine, Shanghai, China. ${ }^{2}$ Department of Gastroenterology, Shanghai Sixth People's Hospital, Shanghai Jiao Tong University School of Medicine, Shanghai, China.

Received: 30 April 2020 Accepted: 25 September 2020

Published online: 02 October 2020

\section{References}

1. Rizvi S, Gores GJ. Pathogenesis, diagnosis, and Management of Cholangiocarcinoma. Gastroenterology. 2013;145(6):10.1053.

2. Cardinale V, Semeraro R, Torrice A, et al. Intra-hepatic and extra-hepatic cholangiocarcinoma: new insight into epidemiology and risk factors. World J Gastrointest Oncol. 2010;2(11):407-16.
3. Welzel TM, McGlynn KA, et al. Impact of classification of hilar cholangiocarcinomas (Klatskin tumors) on the incidence of intra- and extrahepatic cholangiocarcinoma in the United States. J Natl Cancer Inst. 2006:98(12):873-5.

4. Komuta M, Govaere O, Vandecaveye V, et al. Histological diversity in cholangiocellular carcinoma reflects the different cholangiocyte phenotypes. Hepatology. 2012;55(6):1876-88.

5. Cardinale V, Carpino G, Reid L, et al. Multiple cells of origin in cholangiocarcinoma underlie biological, epidemiological and clinical heterogeneity. World J Gastrointest Oncol. 2012;4(5):94-102.

6. Cai X, Zhai J, Kaplan DE, et al. Background progenitor activation is associated with recurrence after hepatectomy of combined hepatocellularcholangiocarcinoma. Hepatology. 2012;56(5):1804-16.

7. Xu M, Xie F, Qian G, et al. Peritumoral ductular reaction: a poor postoperative prognostic factor for hepatocellular carcinoma. BMC Cancer. 2014;14:65.

8. Sobin LH, Gospodarowicz MK, Wittekind C, International Union against Cancer. TNM classification of malignant tumours, 7th ed. Chichester, UK Hoboken, NJ: Wiley-Blackwell; 2010. p 110-114.

9. Scheuer PJ. Classification of chronic viral hepatitis: a need for reassessment. J Hepatol. 1991;13:372-4

10. Weng HL, Feng DC, Radaeva S, et al. IFN- $\gamma$ inhibits liver progenitor cell proliferation in HBV-infected patients and in 3,5-diethoxycarbonyl-1,4dihydrocollidine diet-fed mice. J Hepatol. 2013:59(4):738-45.

11. Palmer WC, Patel T. Are common factors involved in the pathogenesis of primary liver cancers? A meta-analysis of risk factors for intrahepatic cholangiocarcinoma. J Hepatol. 2012;57(1):69-76.

12. Shaib YH, El-Serag HB, Nooka AK, et al. Risk factors for intrahepatic and extrahepatic cholangiocarcinoma: a hospital-based case-control study. Am J Gastroenterol. 2007;102(5):1016-21.

13. Gadd VL, Skoien R, Powell EE, et al. The portal inflammatory infiltrate and ductular reaction in human nonalcoholic fatty liver disease. Hepatology. 2014:59(4):1393-405.

14. Clouston AD, Powell EE, Walsh MJ, et al. Fibrosis correlates with a ductula reaction in hepatitis $C$ : roles of impaired replication, progenitor cells and steatosis. Hepatology. 2005;41(4):809-18

15. Williams MJ, Clouston AD, Forbes SJ. Links between hepatic fibrosis, ductular reaction, and progenitor cell expansion. Gastroenterology. 2014; 146(2):349-56

\section{Publisher's Note}

Springer Nature remains neutral with regard to jurisdictional claims in published maps and institutional affiliations.
Ready to submit your research? Choose BMC and benefit from:

- fast, convenient online submission

- thorough peer review by experienced researchers in your field

- rapid publication on acceptance

- support for research data, including large and complex data types

- gold Open Access which fosters wider collaboration and increased citations

- maximum visibility for your research: over $100 \mathrm{M}$ website views per year

At BMC, research is always in progress.

Learn more biomedcentral.com/submissions 